This is the final peer-reviewed accepted manuscript of:

Manerba M, Di lanni L, Govoni M, Roberti M, Recanatini M, Di Stefano G.

LDH inhibition impacts on heat shock response and induces senescence of hepatocellular carcinoma cells

Eur J Pharm Sci. 2017 Jul 15;105:91-98.

The final published version is available online at:https://doi.org/10.1016/j.ejps.2017.05.015

Rights / License:

The terms and conditions for the reuse of this version of the manuscript are specified in the publishing policy. For all terms of use and more information see the publisher's website. 


\title{
LDH inhibition impacts on heat shock response and induces senescence of hepatocellular carcinoma cells
}

\author{
Marcella Manerba ${ }^{\mathrm{a}}$, Lorenza Di Ianni ${ }^{\mathrm{a}}$, Marzia Govoni ${ }^{\mathrm{a}}$, Marinella Roberti ${ }^{\mathrm{b}}$, Maurizio Recanatini ${ }^{\mathrm{b}}$, \\ Giuseppina Di Stefano ${ }^{\mathrm{a}}$ * \\ ${ }^{\text {a }}$ Department of Experimental, Diagnostic and Specialty Medicine (DIMES), University of Bologna, Italy \\ ${ }^{\mathrm{b}}$ Department of Pharmacy and Biotechnology (FABIT), University of Bologna, Italy
}

\begin{abstract}
A B S T R A C T
In normal cells, heat shock response (HSR) is rapidly induced in response to a variety of harmful conditions and represents one of the most efficient defense mechanism. In cancer tissues, constitutive activation converts HSR into a life-threatening process, which plays a major role in helping cell survival and proliferation. Overexpression of heat shock proteins (HSPs) has been widely reported in human cancers and was found to correlate with tumor progression. Hepatocellular carcinoma is one of the conditions in which HSR activation was shown to have the highest clinical significance.

Transcription of HSPs is induced by HSF-1, which also activates glycolytic metabolism and increases the expression of LDH-A, the master regulator of the Warburg effect. In this paper, we tried to explore the relationship between HSR and LDH-A. In cultured hepatocellular carcinoma cells, by using two enzyme inhibitors (oxamate and galloflavin), we found that the reduction of LDH-A activity led to decreased level and function of the major HSPs involved in tumorigenesis. Galloflavin (a polyphenol) also inhibited the ATPase activity of two of the examined HSPs. Finally, hindering HSR markedly lowered the alpha-fetoprotein cellular levels and induced senescence.

Specific inhibitors of single HSPs are currently under evaluation in different neoplastic diseases. However, one of the effects usually observed during treatment is a compensatory elevation of other HSPs, which decreases treatment efficacy. Our results highlight a connection between LDH and HSR and suggest LDH inhibition as a way to globally impact on this tumor promoting process.
\end{abstract}

\section{Keywords:}

Cancer cell metabolism

Heat shock response

Hepatocellular carcinoma

Lactate dehydrogenase

\section{Introduction}

Heat shock proteins (HSPs) are a family of highly conserved molecular chaperones which help the maintenance of normal protein conformation, protecting the proteome from the dangers of misfolding and aggregation (Morimoto, 1993; Schlesinger, 1990). In normal cells, HSP expression is rapidly induced in response to a wide variety of physiological and environmental insults and was proven to be essential for survival. This protective mechanism was originally discovered by the Italian scientist Ritossa and is usually referred to as "the heat shock response" (HSR) (Ritossa, 1996). It was found to be constitutively activated in cells from several human malignancies: gastroenterological cancers, including hepatocellular carcinoma (HCC), breast, endometrial and squamous carcinomas (Ciocca and Calderwood, 2005). Abnormal
HSR seems to play a critical role in neoplastic change and tumor development, since it protects from degradation proteins that are essential for tumorigenesis and cell proliferation (Calderwood et al., 2006). Moreover, it is believed to contribute to therapeutic resistance and was found to promote tumor invasion and metastasis (Soti and Csemerly, 1998). For these reasons, constitutively activated HSR has been proposed as an independent diagnostic and prognostic marker in cancer patients and is also considered an attractive therapeutic target. Synthetic inhibitors targeting the major members of HSP family (hsp27, hsp72, hsp90) have recently emerged and are under evaluation as novel weapons against neoplastic diseases (Jego et al., 2013).

The major inducer of HSP expression is the transcription factor HSF-1, which is highly activated in cancer cells (Jiang et al., 2015). HSF-1 was also observed to regulate glucose metabolism (Dai et al.,

\footnotetext{
* Corresponding author at: Department of Experimental, Diagnostic and Specialty Medicine (DIMES), Via San Giacomo, 14, 40126 Bologna, Italy.

Email address: giuseppina.distefano@unibo.it (G. Di Stefano)
} 
2007) and to increase the expression of the A isoform of the enzyme lactate dehydrogenase (LDH-A) (Zhao et al., 2009). These findings revealed a connection between the Warburg effect and the HSR. As well known, the Warburg effect is one of the hallmarks of cancer cells and is characterized by enhanced conversion of pyruvate to lactate even in the presence of adequate oxygen levels (Kim and Dang, 2006). This cancer-associated metabolic change is strictly linked to increased expression of LDH-A (Fiume et al., 2014). Moreover, similar to dysregulated HSR, enhanced lactate production by cancer cells was associated with epithelial-mesenchymal transition and with increased capacity of local invasion, the most critical features of cancer lesions (Walenta and Mueller-Klieser, 2004). These findings prompted us to investigate the impact of LDH inhibition on the expression and function of HSPs, focusing our attention on the family members more closely related to human malignancies (hsp27, hsp72, hsp90).

As a model for our study, we chose a cell line (PLC/PRF/5) derived from a human HCC. In spite of recent progress, the prognosis of HCC patients remains poor, because of high rates of tumor recurrence after treatments (Ingle et al., 2016). Accumulating evidence has shown activated HSR as an important modulator of treatment resistance and invasive potential of HCC and upregulation of HSF-1 has been associated with HCC progression (Wang et al., 2016). The three members of HSP family most commonly considered for the design of inhibitors (hsp27, hsp72, hsp90) were repeatedly found to be overexpressed in HCC cells and to play a critical role in apoptosis inhibition and cell survival (Wang et al., 2016). Moreover, promising results have been reported in preclinical studies testing the efficacy of inhibitors (Braconi et al., 2009).
In our experiments, the relationship between HSR and LDH was investigated by hindering the enzyme activity with galloflavin (GF). GF is a derivative of gallic acid identified by our research group during a virtual screening campaign aimed at discovering LDH inhibitors (Manerba et al., 2012). Since the polyphenolic structure of this compound (see Fig. 1) does not allow to exclude additional, not LDH-related, biochemical activities, in all experiments we compared the effects observed with GF with those caused by oxamate (OXA). OXA is the reference LDH inhibitor; it specifically inhibits the enzyme by competing with its natural substrate. Contrary to GF, which is active at micromolar concentrations, OXA is active in the millimolar range (Papacostantinou and Colowick, 1961).

\section{Materials and methods}

\subsection{Cell culture and reagents}

PLC/PRF/5 cells were grown in DMEM ( $1 \mathrm{~g} / 1$ glucose), supplemented with $10 \%$ FBS, $100 \mathrm{U} / \mathrm{ml}$ penicillin/streptomycin, $2 \mathrm{mM}$ glutamine and $1 \mathrm{mM}$ sodium pyruvate. Medium and supplements were from Sigma-Aldrich. During the course of experiments, cells were routinely screened for Mycoplasma contamination and found to be free.

Galloflavin (GF) was synthesized according to the procedure described by Manerba et al. (2012). In all experiments, GF was added to the culture media in the presence of $0.6 \%$ DMSO. The same amount of DMSO was always added to the control, untreated cultures. Unless otherwise specified, all other compounds and all reagents used for the experiments were from Sigma-Aldrich.

\section{Oxamate}

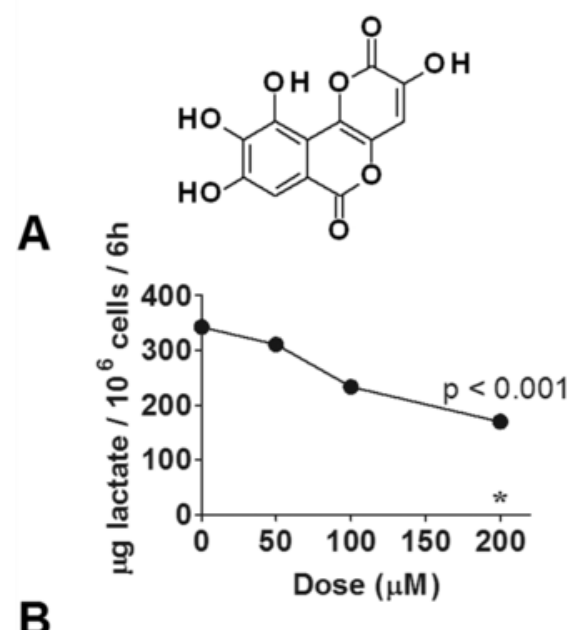

B

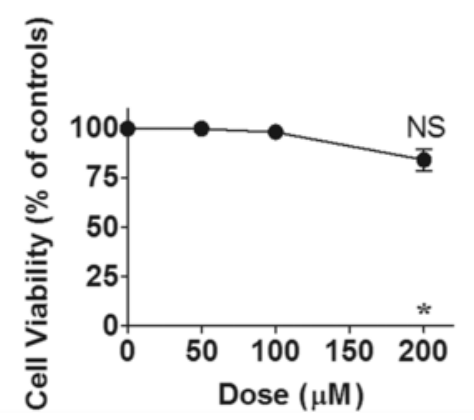<smiles>NC(=O)C(=O)O[Na]</smiles>
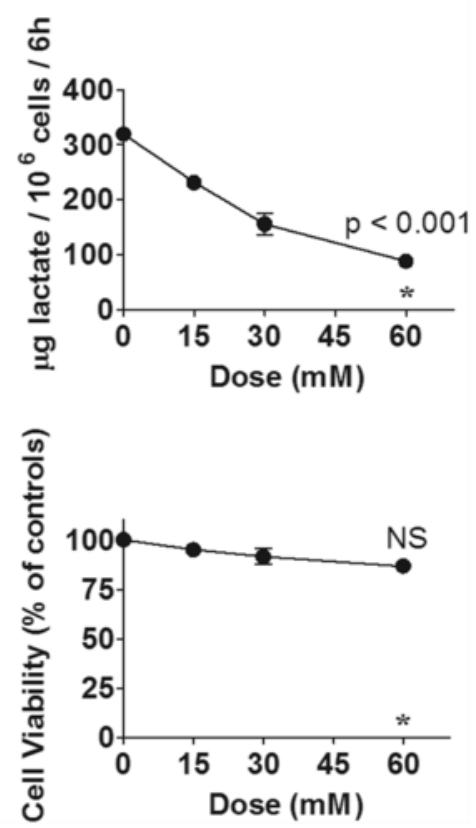

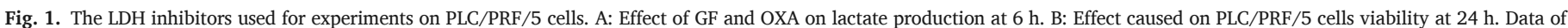

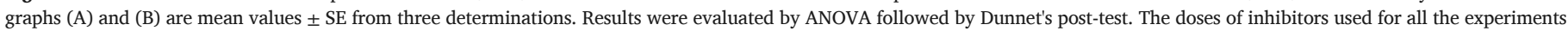
have been marked with an asterisk. They caused statistically significant inhibition of LDH activity, but did not affect cell viability at 24 h. 


\subsection{Lactate and viability assays}

For lactate assay, $5 \times 10^{5}$ cells in $1 \mathrm{ml}$ of culture medium were seeded in each well of a 6-well Nunclon plate and let to adhere overnight. Scalar amounts of OXA $(0-60 \mathrm{mM})$ or GF $(0-\mu \mathrm{M})$ were then added to the cultures. Each dose of compounds was tested in duplicate. The intracellular and released metabolite was measured after $6 \mathrm{~h}$ incubation following the procedure described by Farabegoli et al. (2012).

For the viability assay, cells $\left(1.0 \times 10^{4} /\right.$ well $)$ were seeded in 96 -multiwell plates, allowed to adhere overnight and treated for $24 \mathrm{~h}$ at $37^{\circ} \mathrm{C}$ with OXA and GF (same doses as for the lactate assay, tested in duplicate). After incubation, cells were maintained $3 \mathrm{~h}$ at $37^{\circ} \mathrm{C}$ with the Neutral Red dye dissolved in medium at the final concentration of $30 \mu \mathrm{g} / \mathrm{ml}$. Medium was then removed and the cells were solubilized with $200 \mu \mathrm{l}$ of $1 \%$ acetic acid in $50 \%$ ethanol. Absorbance of the solutions was measured at $\lambda 540$ using a microplate reader (Multiskan Ascent FL, Labsystems).

\subsection{Real time PCR}

HSPs and alpha-fetoprotein (AFP) expressions were evaluated by measuring their mRNA levels, using $\beta$-tubulin and HPRT as internal controls of the reaction. RNA was extracted according to the procedure described by Chomczynski and Sacchi (1987) and was quantified spectrophotometrically. Retro-transcription to cDNA was performed by using the Revert Aid TM First Strand cDNA Synthesis Kit, in different steps: $5 \mathrm{~min}$ denaturation at $65^{\circ} \mathrm{C}, 5 \mathrm{~min}$ annealing at $25^{\circ} \mathrm{C}, 1 \mathrm{~h}$ retro-transcription at $42{ }^{\circ} \mathrm{C}$ and 5 min denaturation at $70{ }^{\circ} \mathrm{C}$. Real Time PCR analysis of cDNA was performed using SYBR Green (SSO Advanced, BioRad). For each protein, the used primers were obtained from published papers, as follows: hsp27 (Tourtas et al., 2012); hsp72 (Walsh et al., 2001); hsp90A (Lauten et al., 2003); hsp90B (Chhabra et al., 2015); AFP (Zhang et al., 2009). Annealing temperature was $60^{\circ} \mathrm{C}$. All samples were run in triplicate, in $10 \mu \mathrm{l}$ reaction volume containing $10 \mathrm{ng}$ of cDNA. The thermal cycler (CFX96 TM Real Time System, BioRad) was programmed as follows: $30 \mathrm{~s}$ at $95^{\circ} \mathrm{C} ; 40$ cycles of $15 \mathrm{~s}$ at $95{ }^{\circ} \mathrm{C} ; 30 \mathrm{~s}$ at $60{ }^{\circ} \mathrm{C}$.

\subsection{2',7'-Dichloro-dihydro-fluorescin diacetate assay}

Cells were grown on cover slips $\left(5 \times 10^{5} /\right.$ well) in 6 -well plates and they were treated for $4 \mathrm{~h}$ with $200 \mu \mathrm{M} \mathrm{GF}$ or $60 \mathrm{mM}$ OXA. After washing with PBS, the cells were incubated at room temperature in the dark for 20 min with $10 \mu \mathrm{M} \mathrm{2}$ ',7'-dichloro-dihydro-fluorescin diacetate (DCFH-DA) dissolved in PBS. After incubation, the cells were rinsed three times with PBS and mounted with a solution of DAPI (300 nM) and DABCO. The samples were observed at a Nikon epifluorescence microscope equipped with filters for DAPI and FITC. At least 3 fields for sample were analyzed and $\geq 200$ cells for sample were counted.

\subsection{Assay of thiobarbituric acid reactive substances (TBARS)}

PLC/PRF/5 cells $\left(6 \times 10^{6}\right)$ were seeded in a T75 flask and let to adhere overnight. They were then exposed for $18 \mathrm{~h}$ to $60 \mathrm{mM}$ OXA or $200 \mu \mathrm{M} \mathrm{GF}$, dissolved in serum free medium. After treatment, they were pelleted and lysed by sonication in $100 \mu \mathrm{l} \mathrm{H}_{2} \mathrm{O}$. Samples were then added with $66 \mu \mathrm{l} 8.1 \%$ SDS, $500 \mu \mathrm{l} 20 \%$ acetic acid pH 3.5, $500 \mu \mathrm{l}$ $0.8 \%$ thiobarbituric acid and heated at $95{ }^{\circ} \mathrm{C}$ for $1 \mathrm{~h}$. After chilling at room temperature, TBARS were extracted with $700 \mu \mathrm{l}$ of 15:1 N-butanol/pyridine. Samples were then centrifuged at $4000 \mathrm{rpm}$ for $10 \mathrm{~min}$ and the absorbance of their organic layer was measured at $\lambda 532$. The amount of TBARS was normalized to the protein content of the cell pellet.

\subsection{ATPase assay}

Recombinant, functionally active hsp72 and hsp90 from Enzo Life Science were used. They were diluted 1:4 to limit interference from their medium (PBS buffer) in the detection of phosphate released by ATPase activity. ATPase activity was measured in a 96-well plate, by using a commercially available kit (Sigma-Aldrich). In each well, $500 \mathrm{ng}$ recombinant protein was added to $30 \mu \mathrm{l}$ assay buffer and incubated $3 \mathrm{~h}$ at $37^{\circ} \mathrm{C}$ with $1 \mathrm{mM}$ GF. $1 \mathrm{mM}$ ATP was then added and the plate was maintained at $37{ }^{\circ} \mathrm{C}$ for further $3 \mathrm{~h}$. Reaction was stopped by adding to each well $200 \mu \mathrm{l}$ of the kit included reagent which, after $20 \mathrm{~min}$ incubation at room temperature generates the colorimetric product. Absorbance was read at $\lambda 620$ by using a microplate reader (Multiskan Ascent FL, Labsystems).

\subsection{Immunoblotting}

Cell cultures were exposed to $60 \mathrm{mM}$ OXA or $200 \mu \mathrm{M}$ GF for $24 \mathrm{~h}$. After incubation, they were lysed in $100 \mu \mathrm{l}$ RIPA buffer containing protease inhibitors. The cell homogenates were left $30 \mathrm{~min}$ on ice and then centrifuged $15 \mathrm{~min}$ at $10000 \mathrm{~g}$. Proteins of the supernatants (40 or $100 \mu \mathrm{g}$, measured according to the method of Bradford) were loaded onto $4-12 \%$ precasted polyacrylamide gels (Life Technologies) for electrophoresis. The separated proteins were blotted on a low fluorescent PVDF membrane (GE Lifescience) using a standard apparatus for wet transfer with an electrical field of $80 \mathrm{~mA}$. The blotted membrane was blocked with 5\% BSA (or casein) in TBS-Tween and probed with the primary antibody. The used antibodies were: rabbit monoclonal anti-AFP (D12C1) (Cell Signaling); mouse monoclonal anti-hsp27 (G31) (Cell Signaling); rabbit anti-phospho-hsp27(Ser78) (Cell Signaling); rabbit anti-hsp90 (Enzo Life Sciences); mouse monoclonal anti-hsp72 (C92F3A-5) (Enzo Life Sciences); rabbit anti-Actin (Sigma Aldrich). Binding was revealed by a Cy5-labelled secondary antibody (anti rabbit-IgG, GE Lifescience; anti mouse-IgG, Jackson Immuno-Research). All incubation steps were performed according to the manufacturer's instructions. Fluorescence of the blots was assayed with the Pharos FX scanner (BioRad) at a resolution of $100 \mu \mathrm{m}$, using the Quantity One software (BioRad). In order to obtain a statistical evaluation of changes in protein levels, all immunoblotting experiments were repeated twice, using samples from two different experimental sets.

\subsection{Senescence-associated $\beta$-galactosidase staining assay}

Cells were plated at a density of $3 \times 10^{4}$ in 6 -well plates. After incubation with $60 \mathrm{mM}$ OXA or $200 \mu \mathrm{M} \mathrm{GF}$ for $48 \mathrm{~h}$, they were fixed in $2 \%$ formaldehyde $/ 0.2 \%$ glutaraldehyde in phosphate-buffered saline for $5 \mathrm{~min}$ and incubated at $37^{\circ} \mathrm{C}$ for 12 to $18 \mathrm{~h}$ with fresh $\beta$-galactosidase staining solution containing $1.0 \mathrm{mg} / \mathrm{ml}$ 5-bromo-4-chloro-3-indolyl- $\beta$-D-galactopyranoside (X-gal). The procedure described by Debacq-Chainiaux et al. (2009) was used.

\subsection{Colony formation assay}

For colony formation assay, cells were plated into 6-well plates, at the density of 500 cells/well. They were treated with $60 \mathrm{mM}$ OXA or $200 \mu \mathrm{M}$ GF (tested in triplicate) for $24 \mathrm{~h}$. Compounds were then removed and cultures were maintained for additional 15 days. Cells were 
stained with $0.5 \%$ crystal violet (dissolved in $6 \%$ glutaraldehyde). The colonies were photographed and counted to generate a histogram.

\subsection{Statistical analyses}

All statistical analyses were performed using the software GraphPad Prism 5. Each experiment was repeated two or three times, with at least triplicate samples per treatment group. Results are expressed as mean \pm SE of replicate values. $\mathrm{p}$ values $<0.05$ were considered statistically significant.

\section{Results}

\section{1. $L D H$ inhibition does not affect $P L C / P R F / 5$ cell viability in the short term $(24 h)$}

As a premise to our experiments, we evaluated the effects of GF and OXA on lactate production and viability of PLC/PRF/5 cells. As shown in Fig. 1A, GF and OXA significantly inhibited lactate production of treated cells. However, contrary to what observed by studying LDH inhibition in other tumor models (Farabegoli et al., 2012; Vettraino et al., 2013), PLC/PRF/ 5 cells appeared to well tolerate the enzyme inhibition, since no statistically significant effects on cell viability were observed after the first $24 \mathrm{~h}$ (Fig. 1B). This result suggests metabolic plasticity by PLC/PRF/5 cultures and is in agreement with previous experiments showing hepatocellular carcinoma cells viability to be significantly affected by OXA and GF only after sustained treatments (Fiume et al., 2010). The ability to adapt to LDH inhibition shown by PLC/PRF/5 cells was essential for the proceeding of the present study, since it allowed to exclude that all the observed effects could be linked to cell death induction. The selected doses of inhibitors used for the study of HSPs have been marked in the graphs of Fig. 1A and B with an asterisk; they are $200 \mu \mathrm{M}$ GF and $60 \mathrm{mM}$ OXA.

\section{2. $L D H$ inhibition and HSPs gene expression}

We evaluated the expression of hsp27, hsp72, hsp90A and hsp90B in PLC/PRF/5 cells exposed to GF and OXA. Hsp90A and B are the hsp90 forms located in the cytoplasm and endoplasmic reticulum, respectively. Fig. 2A shows that GF did not significantly affect the expression of the examined HSPs during the $24 \mathrm{~h}$ treatment; on the contrary, a statistically significant increase of hsp72 and hsp27 was observed during OXA treatment (Fig. 2B). This increase peaked at $18 \mathrm{~h}$, after which it tended to decline. The effects caused by OXA are compatible with a temporary metabolic impairment caused by LDH inhibition, which hinders glycolysis. Hsp72 was already shown to be activated in conditions of metabolic stress and reduced glucose availability when, by improving mitochondrial triage, this chaperone favors oxidative metabolism (Drew et al., 2014; Ludwig et al., 2014). In several tumor models, LDH inhibition was also found to generate oxidative stress (Le et al., 2010; Wang et al., 2012). This finding can give a further explanation to the observed increase of both hsp72 and hsp27. In fact, these chaperones have been described as sensors of cellular redox changes and hsp27 was also found to protect cells from oxidative damage (Kalmar and Greensmith, 2009; Toth et al., 2010).

Taken together, the data of Fig. 2 indicated that LDH inhibition should not induce a hsp90-mediated defense response, since none of the inhibitors affected the expression of both hsp90A and hsp90B. Furthermore, the reported results showed that, in spite of the metabolic impairment produced in treated cells (Fig. 1A), GF did not alter the expression of hsp72 and hsp27. This latest effect is not imputable to LDH inhibition, since it was not displayed by OXA. However, it can be
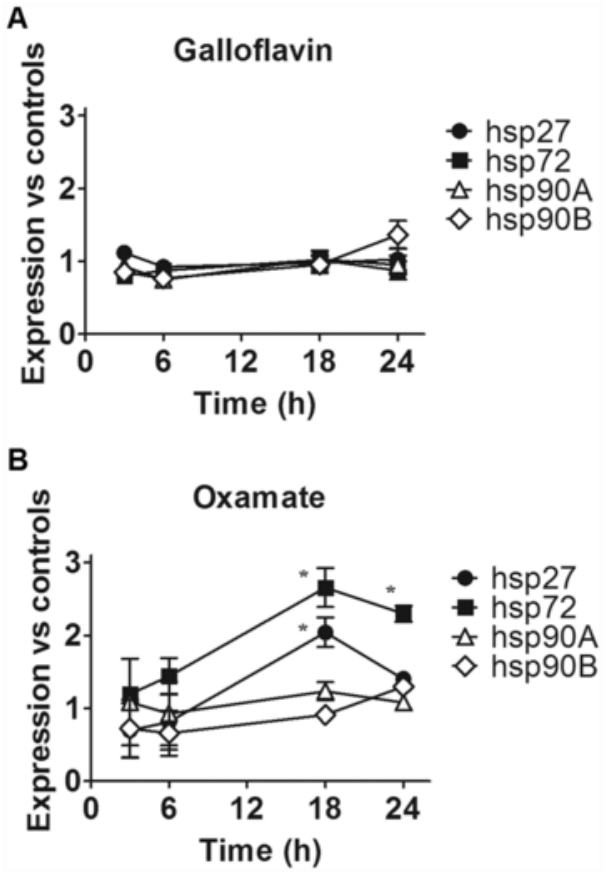

Fig. 2. Expression of hsp27, hsp72 and hsp90 measured by real time PCR in PLC/PRF/5 cells exposed to GF (A) or OXA (B). A: GF did not affect the expression of the three HSPs. B: OXA caused increased hsp27 and hsp72 expression at $18 \mathrm{~h}$, with a subsequent decline at $24 \mathrm{~h}$. *, statistically significant difference compared to control cells, with $\mathrm{p}<0.05$.

viewed as an additional, useful property of the molecule, which could avoid the induction of a defense response potentially helping cancer cell survival. To explain this feature, we hypothesized that, similarly to other phenolic compounds, GF could act as a free radical scavenger (Sureda et al., 2014). A confirmation was obtained by searching signs of oxidative damage in PLC/PRF/5 cells exposed to OXA and GF. Oxidative damage was assessed by evidencing the presence of reactive oxygen species (ROS) and by measuring the level of 2-thiobarbituric acid reactive substances (TBARS). ROS were detected by treating the cells with DCFH-DA. When applied to intact cells, DCFH-DA crosses cell membranes and is enzymatically hydrolyzed by intracellular esterases to non-fluorescent dichlorofluorescin (DCFH). In the presence of ROS, DCFH is oxidized to highly fluorescent dichlorofluorescein (DCF), allowing the detection of ROS production in living cells. DCF-labeled cells can be easily visualized and counted under a fluorescence microscope. The results of DCFH-DA assay are shown in Fig. 3A and B. Fig. 3A shows exemplificative microscopic views of the treated cells. In control cultures and in cultures exposed to OXA or GF, the fraction of DFC labeled cells (right panels) was calculated after counting a total 200-250 cells, evidenced by DAPI staining (left panels). Results have been reported in the graph of Fig. 3B and showed that GF significantly reduced the number of DCF-labeled PLC/PRF/5 cells, when compared to OXA. A further, quantitative evaluation of oxidative stress generation was obtained by measuring TBARS. TBARS are naturally present molecules in biological specimens, usually increasing in concentration as a response to oxidative stress (Armstrong and Browne, 1994). They include lipid hydroperoxides and aldehydes that react with 2-thiobarbituric acid, giving a chromophore. Fig. 3C shows that PLC/PRF/5 cells exposed to GF displayed significantly reduced TBARS staining when compared with control or OXA treated cultures. Both assays confirmed the antioxidant potential of GF and explain why this compound can mitigate the oxidative stress associated with LDH inhibition, preventing the increase of the redox sensitive hsp27 and hsp72. 
A
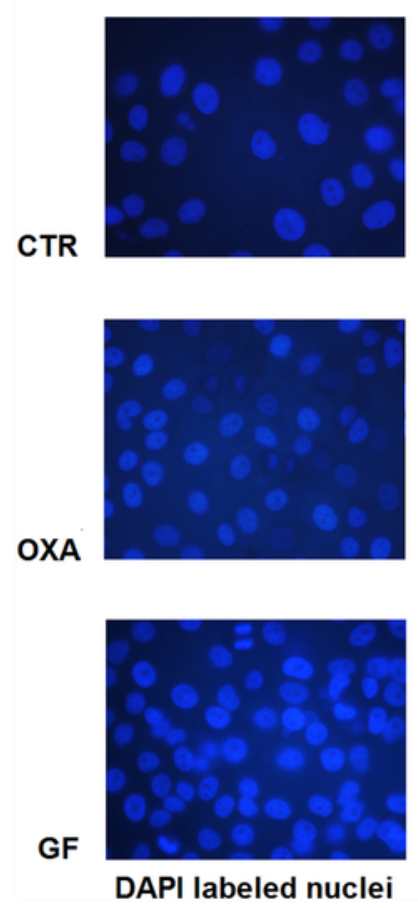

B
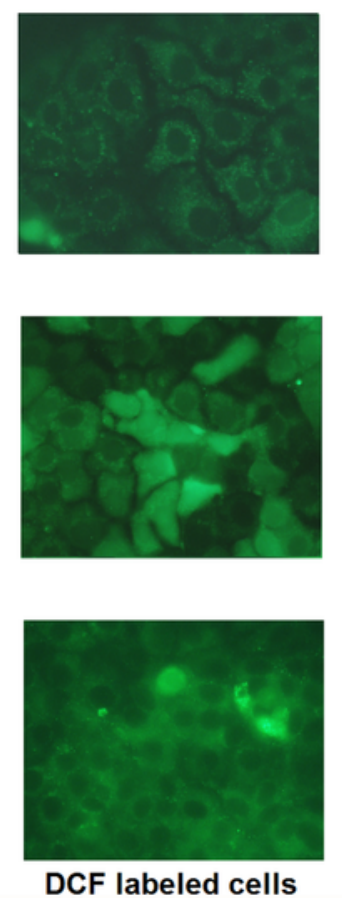

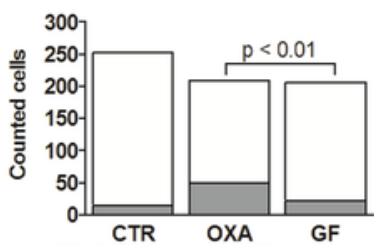

$\square$ DCF-Labeled $\square$ Unlabeled

C

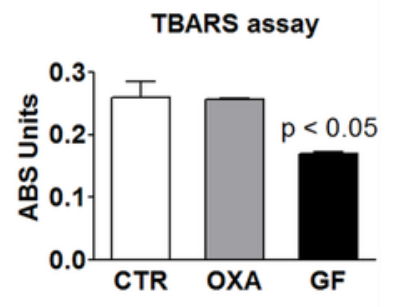

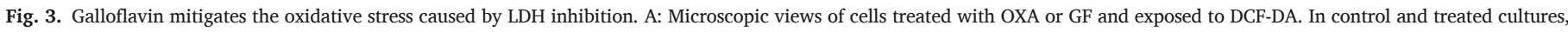

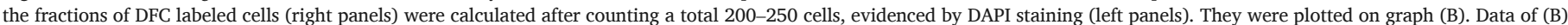

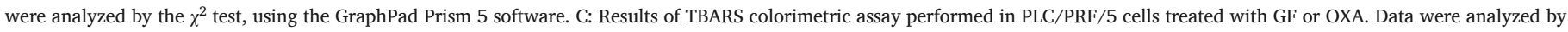
ANOVA followed by Bonferroni post-test.

\subsection{LDH inhibition and HSPs protein level and function}

The levels of HSPs proteins were studied after a $24 \mathrm{~h}$ exposure of PLC/PRF/5 cells to GF and OXA. Fig. 4A shows that OXA significantly decreased all the three chaperones. This decrease was especially evident in the case of hsp27, which became barely detectable, and of hsp90, which was reduced by $60 \%$. Notably, the enhanced expression of both hsp27 and hsp72 observed after $18 \mathrm{~h}$ exposure to OXA (Fig. 2B) did not result in increased protein levels at $24 \mathrm{~h}$, suggesting impairment of mRNA translation presumably linked to LDH inhibition. This hypothesis is in line with previous data showing LDH to be a mRNA binding protein, involved in post-transcriptional regulation of gene expression (Pioli et al., 2002). Fig. 4A also shows that GF generally replicated the effects caused by OXA on HSPs protein levels. The less marked reductions observed with GF could be explained by the slightly lower LDH inhibition power of the compound dose used for the present experiments (see Fig. $1 \mathrm{~A})$.

In addition, we also tried to obtain evidence of the functional status of the three studied HSPs following LDH inhibition. This was easily verified for hsp27, which exerts its chaperone activity in phosphorylated form. Fig. 4A shows that GF treatment did not increase hsp27 phosphorylation, while slightly increased signs of phosphorylation were observed with OXA, which, however, could not be reliably quantified by the densitometric reading of the band. This finding together with the hardly detectable level of hsp27 in OXA treated cells suggest that LDH inhibition could markedly compromise the chaperone activity of this protein.

Contrary to hsp27, hsp72 and hsp90 exert their function through intrinsic ATPase activity. Published studies showed that myricetin, a natural flavonoid very similar to GF in its molecular structure, inhibits the ATPase activity of hsp70 and related proteins (Chang et al., 2011). Molecular docking studies hypothesized the binding of myricetin to a site close to the nucleotide-binding cleft of hsp70, which could explain the loss of enzymatic activity. Flavonoids and related compounds were also found to inhibit the function of hsp90 (Davenport et al., 2014). On these bases, we developed an in vitro ATPase assay using human recombinant hsp72 and hsp90 and evaluated whether GF can hinder the enzymatic activity of the two chaperones. Results are reported in Fig. 4B and showed for both HSPs a statistically significant reduction of ATPase function, which was almost completely abolished for hsp72.

Taken together, these results suggest that LDH inhibition can hamper the dysregulated HSR of HCC cells, since it significantly reduced the protein level of the major HSPs. The functional study of HSPs confirmed that GF can affect HSR not only through LDH inhibition, but also thanks to other properties of its molecular structure. Compared to OXA, more evident effects related to HSR inhibition can be expected by using this compound.

\subsection{Study of cell senescence}

The chaperones most heavily affected by GF treatment (hsp27 and hsp72) have been described as critical factors in apoptosis resistance and replicative potential of cancer cells (Jego et al., ; Wang et al., 2016). Hsp72 was also found to regulate the level of alpha-fetoprotein (AFP) the chief marker of HCC development (Wang et al., 2013). To verify whether the impaired HSR caused by GF and OXA can limit the replicative capacity of PLC/PRF/5 cells, we evaluated $\beta$-galactosidase activity by staining the treated cells with the chromogenic substrate 5-bromo-4-chloro-3-indolyl- $\beta$-D-galactopyranoside (X-gal). The detection of this enzymatic activity is a widely-used procedure for assessing induction of senescence in mammalian cells (Debacq-Chainiaux et al., 2009). Results are shown in Fig. 5A. The $\beta$-galactosidase staining, which scantily appeared in the control culture, became evident in cells treated with both LDH inhibitors, showing bright intensity especially in cells treated with GF. Since a quantitative assessment is not easily 
A

Actin

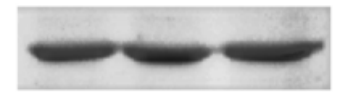

Hsp72

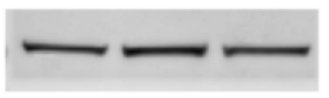

Hsp90

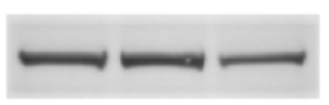

Hsp27

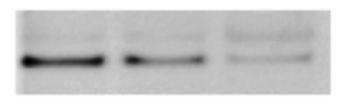

Phospho-

Hsp27
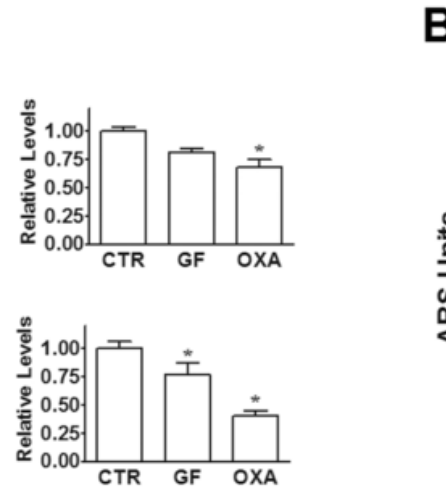

B

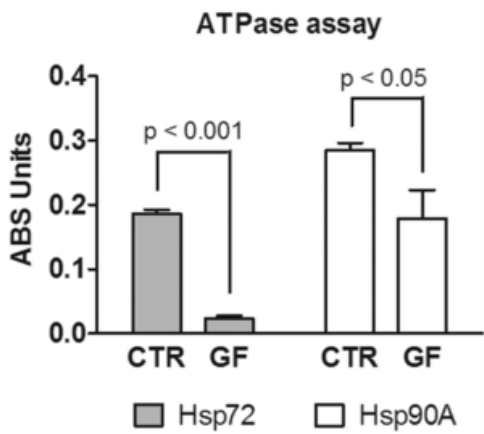

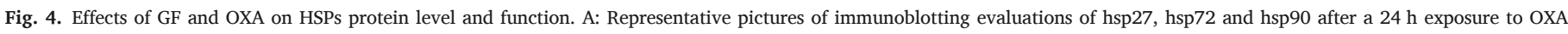

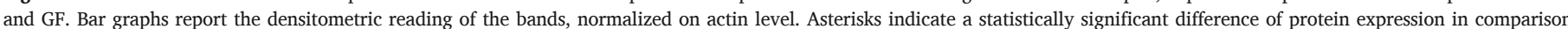

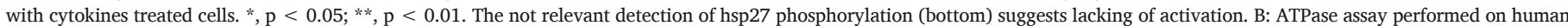

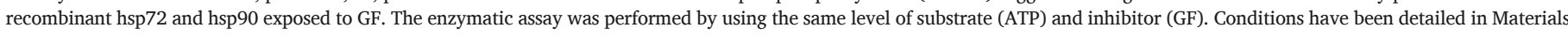
and Methods.

A

\section{$\beta$ - Galactosidase Staining}

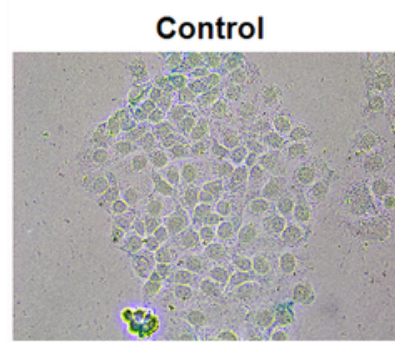

B
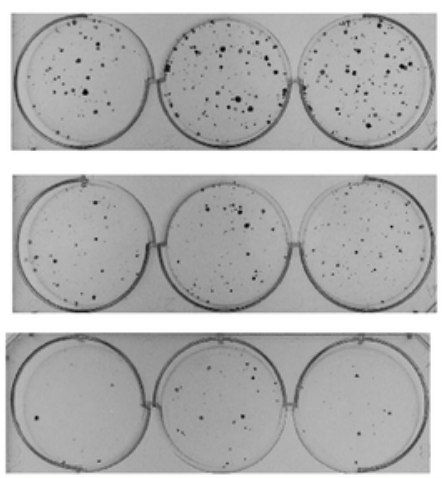

Oxamate

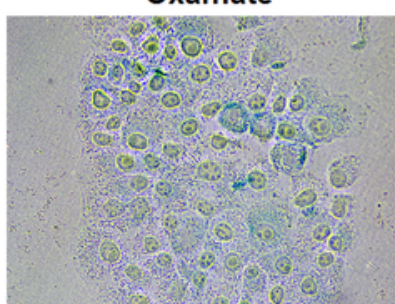

Clonogenicity

\section{Control}

Oxamate

Galloflavin

\section{Number of Colonies}

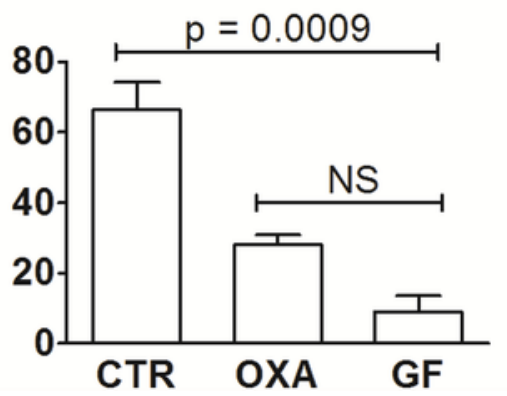

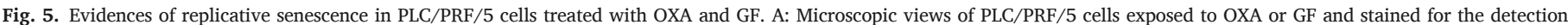

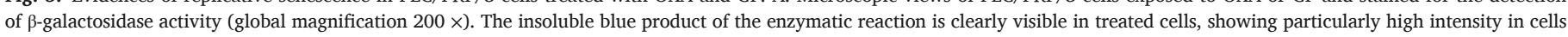

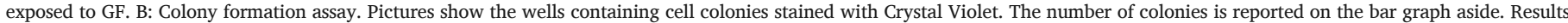
were evaluated by ANOVA followed by Bonferroni post-test. 
achievable from this experiment, we added a further evaluation by performing a clonogenicity assay, which allows to easily count the number of visible colonies formed by plated cells after treatment.

Fig. 5B shows photographs of the cell cultures at the end of the assay and the number of counted colonies, plotted on a bar graph. ANOVA followed by Bonferroni post-test indicated a statistically significant difference between treated and control cells; again, GF apparently proved to be more efficient in reducing proliferative potential, although statistical evaluation indicated no significant difference in comparison with OXA.

In HCC cells, proliferative potential and resistance to apoptosis correlate with the level of AFP, which was found to significantly decrease expression of the cyclin dependent kinase inhibitor p27 and to increase proliferating cell nuclear antigen (PCNA) (Terentiev and Moldogazieva, 2013). Results shown in Fig. 6 support and explain the findings described in Fig. 5. Both OXA and GF caused a sharp reduction in AFP level of treated cells (Fig. 6B). Interestingly, when the levels of mRNA were examined, we found no effect with OXA, and a marked, statistically significant reduction with GF (Fig. 6A). This finding suggests that the effects caused by OXA on AFP could be mediated by the impaired HSR, since they occur at protein but not at mRNA level. The data of Fig. $6 \mathrm{~B}$ also confirm additional properties for GF, which amplify the effects of this molecule on HSR and cell senescence.

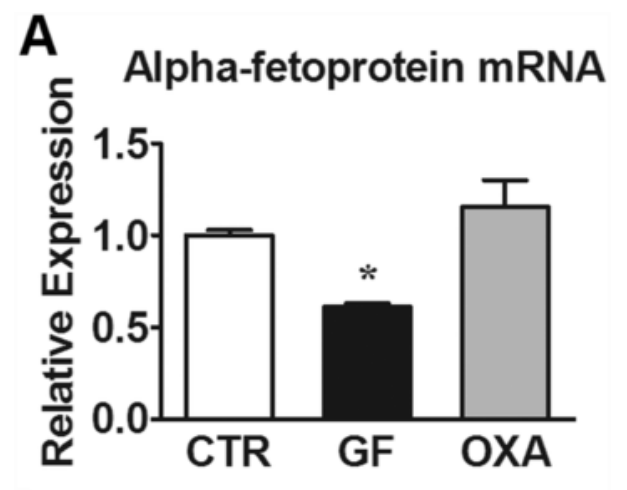

B


Fig. 6. Effect of LDH inhibitors on alpha-fetoprotein expression and level. A: Real time PCR evaluation of AFP expression after a $24 \mathrm{~h}$ exposure to OXA and GF. Contrary to OXA, GF significantly reduced AFP mRNA level. Asterisk indicates a statistically significant difference compared to control and OXA-treated cells, with $\mathrm{p}<0.05$. B: Both LDH inhibitors significantly reduced the protein level. **, $\mathrm{p}<0.01$ compared to control cells.

\section{Discussion}

By protecting cells from a variety of harmful conditions, HSR represents one of the most efficient cellular defense mechanism, but it converts into a double-edge sword in cancer cells, where it becomes constitutively activated and preserves from degradation proteins necessary for survival (Jiang et al., 2015). For these reasons, HSPs have been regarded as novel targets for anticancer therapy and the search of inhibitors has dramatically increased over the last few years (Jego et al., 2013). The two high molecular weight chaperones require ATP for their function and the ATP binding site has been considered for the design of molecules hindering the ATPase activity. A number of hsp72 and hsp90 inhibitors have been tested both on cultured cancer cells and in clinical trials (Braconi et al., 2009; Jego et al., 2013). Recently, AUY922 (a third generation hsp90 inhibitor) was also studied in patients with HCC and showed therapeutic potential (Cheng et al., 2015). However, one of the effects observed during the treatment with this and other hsp90 inhibitors is a compensatory elevation of hsp72, which is finally expected to decrease the efficacy of the anticancer treatment (Jego et al., 2013). For this reason, combined therapies using inhibitors of both the chaperones are currently under consideration.

On the other hand, the low molecular weight hsp27 does not display ATPase activity, but requires phosphorylation to exert its function, which makes it not amenable for inhibition by small molecules. The most studied hsp27 inhibitor is a second-generation antisense oligonucleotide (OGX-427) which reduces protein expression and is now under evaluation in clinical trials (Chi et al., 2016).

The data of our experiments evidenced a connection between HSR and LDH-A, which is supported by the finding that HSF-1 (the master regulator of HSPs transcription) was also shown to induce the transcription of the LDH-A gene (Zhao et al., 2009). In the studied cellular model, LDH inhibition by OXA was found to impact on constitutively activated HSR by reducing the levels of the three examined HSPs, which resulted in cell senescence. The most impressive results concerned hsp27, which became hardly detectable and did not show appreciable signs of activation (phosphorylation). This finding acquires relevance in light of recent studies showing hsp27 to be significantly increased in patients with HCC compared to healthy people or individuals with chronic HBV infection, suggesting hsp27 as a potential biomarker for HCC diagnosis (Feng et al., 2005; Gruden et al., 2013). Moreover, the level of this molecular chaperone was found to have prognostic value, since it is related to apoptosis resistance and metastatic potential (Jego et al., 2013).

GF inhibits LDH activity but, similarly to other polyphenols (Colic and Pavelic, 2000), possess multiple biochemical properties. Besides reproducing OXA effects, GF was also found to hinder the ATPase activity of hsp72 and hsp90 and to be a more efficient inducer of cell senescence.

Taken together, our results suggest that LDH inhibition could be an efficient way to reduce the constitutively activated HSR in cancer cells, since differently from the currently available HSPs inhibitors, it can hinder at the same time the function of all the three major molecular chaperones involved in tumorigenesis. Moreover, the data obtained with GF suggest that, for its peculiar properties, the polyphenolic structure could be an appropriate molecular scaffold to design compounds joining LDH inhibitory activity with other anticancer effects. The interesting pharmacological properties of polyphenols, combined with their good tolerability for normal cells, are now encouraging studies aimed at finding appropriate formulations to overcome the reduced solubility and bioavailability of these compounds (Gao and $\mathrm{Hu}, 2010$; Lewandowska et al., 2013).

Finally, by evidencing its connection with the HSR, our experiments propose a new way of conceiving LDH-A: universally considered a reg- 
ulator of cancer cell metabolism and a factor promoting the active growth of cancer cells (Fiume et al., 2014), this protein could be more appropriately viewed as a defense element which facilitates cell survival.

\section{Conflict of interest}

The Authors have no conflict of interest to declare.

\section{Acknowledgements}

This work was supported by Cornelia and Roberto Pallotti Legacy for Cancer Research and by a grant from Italian Ministry of University and Research (MIUR) (PRIN2012 prot. 2012ZHN9YH).

\section{References}

Armstrong, D., Browne, R., 1994. The analysis of free radicals, lipid peroxides, antioxidant enzymes and compounds related to oxidative stress as applied to the clinical chemistry laboratory. Adv. Exp. Med. Biol. 366, 43-58.

Braconi, C., Meng, F., Swenson, E., Khrapenko, L., Huang, N., Patel, T., 2009. Candidate therapeutic agents for hepatocellular cancer can be identified from phenotype-associated gene expression signatures. Cancer 115, 3738-3748.

Calderwood, S.K., Khaleque, M.A., Sawyer, D.B., Ciocca, D.R., 2006. Heat shock proteins in cancer: chaperones of tumorigenesis. Trends Biochem. Sci. 31, 164-172.

Chang, L., Miyata, Y., Ung, P.M., Bertelsen, E.B., McQuade, T.J., Carlson, H.A., Zuiderweg, E.R., Gestwicki, J.E., 2011. Chemical screens against a reconstituted multiprotein complex: myricetin blocks DnaJ regulation of DnaK through an allosteric mechanism. Chem. Biol. 18, 210-221.

Cheng, W., Ainiwaer, A., Xiao, L., Cao, Q., Wu, G., Yang, Y., Mao, R., Bao, Y., 2015. Role of the novel HSP90 inhibitor AUY922 in hepatocellular carcinoma: potential for therapy. Mol. Med. Rep. 12, 2451-2456.

Chhabra, S., Jain, S., Wallace, C., Hong, F., Liu, B., 2015. High expression of endoplasmic reticulum chaperone grp94 is a novel molecular hallmark of malignant plasma cells in multiple myeloma. J. Hematol. Oncol. 8, 77.

Chi, K.N., Yu, E.Y., Jacobs, C., Bazov, J., Kollmannsberger, C., Higano, C.S., Mukherjee, S.D., Gleave, M.E., Stewart, P.S., Hotte, S.J., 2016. A phase I dose-escalation study of apatorsen (OGX-427), an antisense inhibitor targeting heat shock protein 27 (Hsp27), in patients with castration-resistant prostate cancer and other advanced cancers. Ann. Oncol. 27, 1116-1122.

Chomczynski, P., Sacchi, N., 1987. Single-step method of RNA isolation by acid guanidinium thiocyanate-phenol-chloroform extraction. Anal. Biochem. 162, 156-159.

Ciocca, D.R., Calderwood, S.K., 2005. Heat shock proteins in cancer: diagnostic, prognostic, predictive, and treatment implications. Cell Stress Chaperones 10, 86-103.

Colic, M., Pavelic, K., 2000. Molecular mechanisms of anticancer activity of natural dietetic products. J. Mol. Med. 78, 333-336.

Dai, C., Whitesell, L., Rogers, A.B., Lindquist, S., 2007. Heat shock factor 1 is a powerful multifaceted modifier of carcinogenesis. Cell 130, 1005-1018.

Davenport, J., Balch, M., Galam, L., Girgis, A., Hall, J., Blagg, B.S., Matts, R.L., 2014. High-throughput screen of natural product libraries for hsp90 inhibitors. Biol. (Basel) 3, 101-138.

Debacq-Chainiaux, F., Erusalimsky, J.D., Campisi, J., Toussaint, O., 2009. Protocols to detect senescence-associated beta-galactosidase (SA-betagal) activity, a biomarker of senescent cells in culture and in vivo. Nat. Protoc. 4, 1798-1806.

Drew, B.G., Ribas, V., Le, J.A., Henstridge, D.C., Phun, J., Zhou, Z., Soleymani, T., Daraei, P., Sitz, D., Vergnes, L., Wanagat, J., Reue, K., Febbraio, M.A., Hevener, A.L., 2014. HSP72 is a mitochondrial stress sensor critical for parkin action, oxidative metabolism, and insulin sensitivity in skeletal muscle. Diabetes 63, 1488-1505.

Farabegoli, F., Vettraino, M., Manerba, M., Fiume, L., Roberti, M., Di Stefano, G., 2012. Galloflavin, a new lactate dehydrogenase inhibitor, induces the death of human breast cancer cells with different glycolytic attitude by affecting distinct signaling pathways. Eur. J. Pharm. Sci. 47, 729-738.

Feng, J.T., Liu, Y.K., Song, H.Y., Dai, Z., Qin, L.X., Almofti, M.R., Fang, C.Y., Lu, H.J., Yang, P.Y., Tang, Z.Y., 2005. Heat-shock protein 27: a potential biomarker for hepatocellular carcinoma identified by serum proteome analysis. Proteomics 5, 4581-4588.

Fiume, L., Manerba, M., Vettraino, M., Di Stefano, G., 2010. Impairment of aerobic glycolysis by inhibitors of lactic dehydrogenase hinders the growth of human hepatocellular carcinoma cell lines. Pharmacology 86, 157-162.

Fiume, L., Manerba, M., Vettraino, M., Di Stefano, G., 2014. Inhibition of lactate dehydrogenase activity as an approach to cancer therapy. Future Med. Chem. 6, 429-445.

Gao, S., Hu, M., 2010. Bioavailability challenges associated with development of anti-cancer phenolics. Mini-Rev. Med. Chem. 10, 550-567.

Gruden, G., Carucci, P., Lolli, V., Cosso, L., Dellavalle, E., Rolle, E., Cantamessa, A., Pinach, S., Abate, M.L., Campra, D., Brunello, F., Bruno, G., Rizzetto, M., Perin, P.C.,
2013. Serum heat shock protein 27 levels in patients with hepatocellular carcinoma. Cell Stress Chaperones 18, 235-241.

Ingle, P.V., Samsudin, S.Z., Chan, P.Q., Ng, M.K., Heng, L.X., Yap, S.C., Chai, A.S., Wong, A.S., 2016. Development and novel therapeutics in hepatocellular carcinoma: a review. Ther. Clin. Risk Manag. 12, 445-455.

Jego, G., Hazoumé, A., Seigneuric, R., Garrido, C., 2013. Targeting heat shock proteins in cancer. Cancer Lett. 332, 275-285.

Jiang, S., Tu, K., Fu, Q., Schmitt, D.C., Zhou, L., Lu, N., 2015. Multifaceted roles of HSF1 in cancer. Tumor Biol. 36, 4923-4931.

Kalmar, B., Greensmith, L., 2009. Induction of heat shock proteins for protection against oxidative stress. Adv. Drug Deliv. Rev. 61, 310-318.

Kim, J.W., Dang, C.V., 2006. Cancer's molecular sweet tooth and the Warburg effect. Cancer Res. 66, 8927-8930.

Lauten, M., Beger, C., Gerdes, K., Asgedom, G., Kardinal, C., Welte, K., Schrappe, M., 2003. Expression of heat shock protein 90in glucocorticoid-sensitive and -resistant childhood acute lymphoblastic leukemia. Leukemia 17, 1551-1556.

Le, A., Cooper, C.R., Gouw, A.M., Dinavahi, R., Maitra, A., Deck, L.M., Royer, R.E., Vander Jagt, D.L., Semenza, G.L., Dang, C.V., 2010. Inhibition of lactate dehydrogenase A induces oxidative stress and inhibits tumor progression. Proc. Natl. Acad. Sci. U. S. A. $107,2037-2042$.

Lewandowska, U., Szewczyk, K., Hrabec, E., Janecka, A., Gorlach, S., 2013. Overview of metabolism and bioavailability enhancement of polyphenols. J. Agric. Food Chem. 61, 12183-12199.

Ludwig, M.S., Minguetti-Camara, V.C., Heck, T.G., Pizzato Scomazzon, S., Renck Nunes, P., Barbosa Bazotte, R., Homem de Bittencourt Jr., P.I., 2014. Short-term but not long-term hypoglycaemia enhances plasma levels and hepatic expression of HSP72 in insulin-treated rats: an effect associated with increased IL-6 levels but not with IL-10 or TNF- $\alpha$. Mol. Cell. Biochem. 397, 79-107.

Manerba, M., Vettraino, M., Fiume, L., Di Stefano, G., Sartini, A., Giacomini, E., Roberti, M., Recanatini, M., 2012. Galloflavin (CAS 568-80-9), a novel inhibitor of lactate dehydrogenase. ChemMedChem 7, 311-317.

Morimoto, L.I., 1993. Cells in stress: transcriptional activation of heat shock genes. Science 259, 1409-1410.

Papacostantinou, J., Colowick, S.P., 1961. The role of glycolysis in the growth of tumor cells. I. Effects of oxamic acid on the metabolism of Ehrlich tumor cells in vitro. J. Biol. Chem. 236, 278-284.

Pioli, P.A., Hamilton, B.J., Connolly, J.E., Brewer, G., Rigby, W.F., 2002. Lactate dehydrogenase is an AU-rich element-binding protein that directly interacts with AUF1. J. Biol. Chem. 277, 35738-35745.

Ritossa, F., 1996. Discovery of the heat shock response. Cell Stress Chaperones 1, 97-98.

Schlesinger, M.J., 1990. Heat shock proteins. J. Biol. Chem. 265, 12111-12114.

Soti, C., Csemerly, P., 1998. Molecular chaperones in the etiology and therapy of cancer. Pathol. Oncol. Res. 4, 316-321.

Sureda, A., Tejada, S., Bibiloni Mdel, M., Tur, J.A., Pons, A., 2014. Polyphenols: well beyond the antioxidant capacity: polyphenol supplementation and exercise-induced oxidative stress and inflammation. Curr. Pharm. Biotechnol. 15, 373-379.

Terentiev, A.A., Moldogazieva, N.T., 2013. Alpha-fetoprotein: a renaissance. Tumor Biol. 34, 2075-2091.

Toth, M.E., Gonda, S., Vigh, L., Santha, M., 2010. Neuroprotective effect of small heat shock protein, Hsp27, after acute and chronic alcohol administration. Cell Stress Chaperones 15, 807-817.

Tourtas, T., Birke, M.T., Kruse, F.E., Welge-Luseen, U.C., Birke, K., 2012. Preventive effects of omega- 3 and omega- 6 fatty acids on peroxide mediated oxidative stress responses in primary human trabecular meshwork cells. PLoS One 7, e31340.

Vettraino, M., Manerba, M., Govoni, M., Di Stefano, G., 2013. Galloflavin suppresses lactate dehydrogenase activity and causes MYC downregulation in Burkitt lymphoma cells through NAD/NADH-dependent inhibition of sirtuin-1. Anti-Cancer Drugs 24, 862-870.

Walenta, S., Mueller-Klieser, W.F., 2004. Lactate: mirror and motor of tumor malignancy. Semin. Radiat. Oncol. 14, 267-274.

Walsh, R.C., Koukoulas, I., Garnham, A., Moseley, P.L., Hargreaves, M., Febbraio, M.A., 2001. Exercise increases serum hsp72 in humans. Cell Stress Chaperones 6, 386-393.

Wang, Z.Y., Loo, T.Y., Shen, J.G., Wang, N., Wang, D.M., Yang, D.P., Mo, S.L., Guan, X.Y., Chen, J.P., 2012. LDH-A silencing suppresses breast cancer tumorigenicity through induction of oxidative stress mediated mitochondrial pathway apoptosis. Breast Cancer Res. Treat. 131, 791-800.

Wang, X., Wang, Q., Lin, H., Li, S., Sun, L., Yang, Y., 2013. HSP72 and gp96 in gastroenterological cancers. Clin. Chim. Acta 417, 73-79.

Wang, C., Zhang, Y., Guo, K., Wang, N., Jin, H., Liu, Y., Qin, W., 2016. Heat shock proteins in hepatocellular carcinoma: molecular mechanism and therapeutic potential. Int. J. Cancer 138, 1824-1834.

Zhang, Y.N., Lie, P.C., Wei, X., 2009. Differentiation of mesenchymal stromal cells derived from umbilical cord Wharton's jelly into hepatocyte-like cells. Cytotherapy $11,548-558$.

Zhao, Y.H., Zhou, M., Liu, H., Ding, Y., Khong, H.T., Yu, D., Fodstad, O., Tan, M., 2009 Upregulation of lactate dehydrogenase A by ErbB2 through heat shock factor 1 promotes breast cancer cell glycolysis and growth. Oncogene 28, 3689-3701. 